\title{
Effect of Direct Fed Microbial Supplementation in Late Lay Hen Egg Production and Egg Quality
}

\section{DOI:10.31274/air.13903}

\author{
Claudia DeLeon, Graduate Research Assistant; \\ Maddison Wiersema, Graduate Research Assistant; \\ Dawn Koltes, Assistant Professor; \\ Department of Animal Science, Iowa State University
}

\section{Summary and Implications}

Egg production and quality decrease with hen age and/or prolonged production cycles. Strategies to prevent this decline are important as it allows egg producers to continue to produce a safe and high-quality product for consumers while keeping costs minimal. One strategy to improve egg production and egg quality during late lay is through feed supplementation. This study explored supplementation of late lay (60 + weeks of age) hens with a Bacillus subtilis strain in the feed. Compared to hens that remained on the non-supplemented feed from the same flock, hens supplemented with Bacillus subtilis had increased egg production as measured by hen day egg production and egg weight. However, supplementation did not alter hen mortality, body weight, feed conversion ratio, and egg quality.

\section{Introduction}

When hens age, overall egg production declines, and egg quality traits such as eggshell strength decrease, resulting in fewer marketable eggs for a producer. Identification of strategies that are consumer-accepted to improve the number of marketable eggs during the latter part of the production curve can be economically beneficial to the producer.

Direct fed microbials are common feed additives that are utilized by many sectors in the livestock industry to improve performance, presumably through improved intestinal health. In poultry, meat bird producers have increased yield as a result of bringing more birds to market and increased body weight. However, fewer studies have examined the effects of direct fed microbials on egg production and egg quality in laying hens. Therefore, this study was conducted to determine the effects of adding a Bacillus subtilis strain (Q-Biotic 1DP, Quality Technology International) direct fed microbial in the feed of laying hens starting at 60 weeks of age to determine the effects on hen performance, egg production, and egg quality through 75 weeks of age.

\begin{abstract}
Materials and Methods
Hy-Line W-80 pullets were placed in group cages at 18 weeks of age at the Robert T. Hamilton Poultry Teaching and Research farm in March of 2020. All hens were housed in a single room and were fed a similar diet. At 60 weeks of age, hens were placed in 48 cages with six hens per cage $(n=288)$ Performance and egg quality data were collected from 60 to 62 weeks of age, to determine baseline measurements. Starting at 63 weeks of age, half of the cages were switched to the baseline diet with the addition of a DFM of Bacillus subtilis strain (Q-Biotic 1DP; Quality Technology International), while the remaining cages were maintained on the baseline diet. The hens fed these diets for 12 weeks. Hen performance was measured throughout both the baseline and experimental periods, by recording daily egg production, egg weight, and hen mortality; weekly body weight was recorded, and hen-day egg production was calculated. Egg quality, eggshell strength, Haugh unit, and yolk color were determined using a DET6000 three days per week. Data were analyzed for the experimental period using a generalized linear model with dietary treatment, hen age, and the interaction between dietary and age fit as fixed effects, and the baseline period fit as a covariate.
\end{abstract}

\section{Results and Discussion}

Hen mortality $(\mathrm{P}>0.68)$ and hen body weight $(\mathrm{P}>$ 0.24 ) were similar for both dietary treatment groups during the experimental period (Figure 1). Average hen day egg production (Figure 2) decreased over time; however, hens supplemented with the DFM had a greater hen day egg production by $1.78 \%$ in comparison to hens fed only the baseline diet $(\mathrm{P}<0.04)$. As expected with age, egg weight increased as hens aged $(\mathrm{P}<0.01)$. Supplementation with the DFM resulted in greater individual egg weights compared to the baseline diet by $0.5 \mathrm{~g}(0.02 \mathrm{oz})$ on average $(\mathrm{P}<0.01)$. Although these differences may seem small, when considered at production levels, the increase of $1.78 \%$ on a flock of 1 million hens results in 17,800 eggs per day. In addition, these eggs yielded a $0.5 \mathrm{~g}(0.02 \mathrm{oz})$ increase in weight. On the same 1-million hen flock with a hen day egg production percent of $87 \%$ (the average of our two diets over the experimental period), this would result in $43.5 \mathrm{~kg}$ $(95.7 \mathrm{lb})$ of egg per day.

Several egg quality traits, including eggshell thickness, eggshell strength, Haugh unit, and yolk color, were measured on all eggs laid on three consecutive days each week. Eggshell thickness declined as hens aged $(\mathrm{P}<0.01)$ and was similar between dietary treatments $(\mathrm{P}>0.95)$.

Copyright (9) 2022 by the Authors. This is an open access article published under the CC BY-NC license (https://creativecommons.org/licenses/by-nc/4.0/), which allows for non-commercial reuse with proper attribution. 
Average eggshell thickness ranged between 0.40 and 0.37 $\mathrm{mm}$, which is within the acceptable eggshell thickness range. When examining eggshell strength, it declined as hens aged $(\mathrm{P}<0.01)$ and was greater in hens fed the baseline feed $(\mathrm{P}<0.04)$. This decline in eggshell strength was not unexpected because eggshell strength is negatively associated with egg size. Additionally, eggshell strength is within acceptable ranges. For internal egg quality parameters, Haugh unit declined as birds aged $(\mathrm{P}>0.01)$, although measurements were not different by dietary treatment $(\mathrm{P}>0.37)$. Egg yolk color became more orange as hens aged $(P>0.01)$ and was more orange in eggs from hens fed the baseline diet $(\mathrm{P}<0.02)$; however, these changes were very small $(0.7 \%)$ and not likely detectable to a consumer's eye. Overall, external and internal egg quality minimally changed and were maintained within acceptable ranges; therefore, the advantages observed in hen performance did not decrease the overall egg quality, indicating the use of DFM may be a strategy to improve late lay hen performance but not at the cost of the egg quality.

\section{Acknowledgements}

We gratefully acknowledge the donation of Q-Biotic 1DP by Quality Technology International, laying hens by Hy-Line North America, and pullet rearing by the Iowa Egg Council. We additionally thank the staff of the Robert T. Hamilton Research and Teaching farm for their assistance with the layer flock and this research trial. Funding for this project was provided by the Department of Animal Science, College of Agriculture and Life Sciences, and Iowa State University.. 
A.

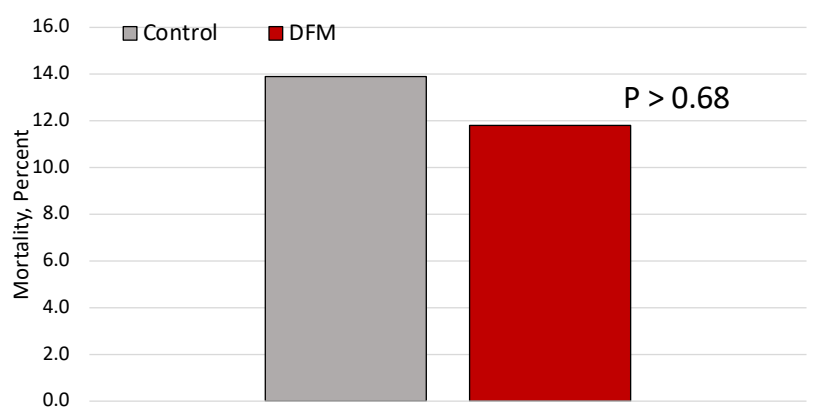

B. 6

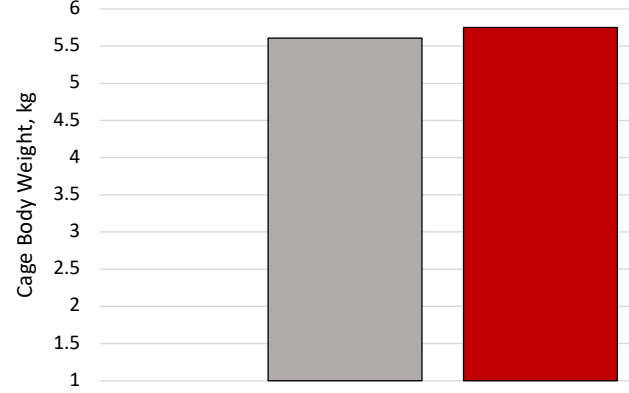

$P>0.28$

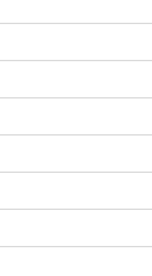

Figure 1. Hen performance throughout the experimental period. A. Hen mortality, B. Hen body weight by cage (n=6 hens/cage). Grey bars represent hens fed the baseline or control diet. Red bars represent hens fed the DFM supplemented diet.

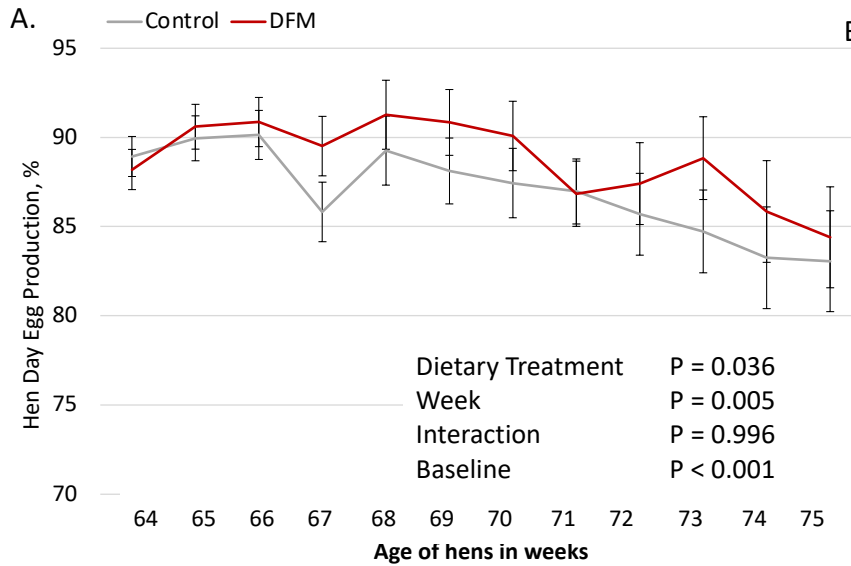

B. 62

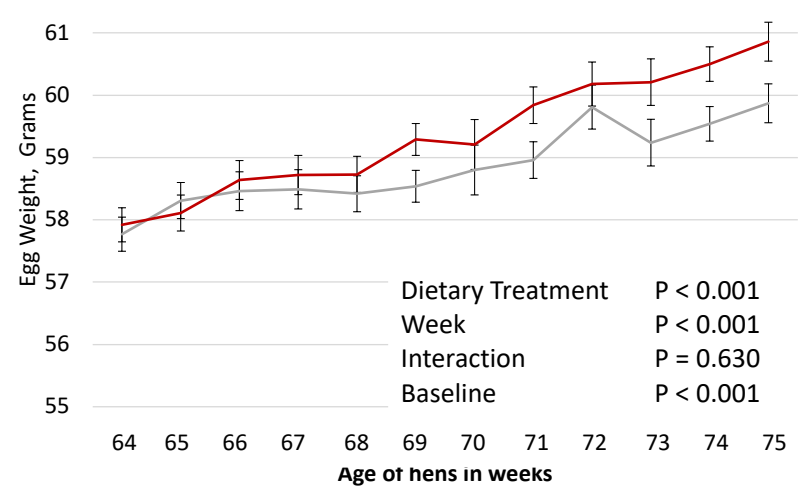

Figure 2. Egg production and average individual egg weight throughout the experimental period. A. Weekly hen day egg production data which is egg production accounting for hen number (total number of eggs produced divided by hen days over a seven day period multiple by 100). B. The average egg weight of individual eggs produced. Grey lines represent hens fed the baseline or control diet. Red lines represent hens fed the DFM supplemented diet. 


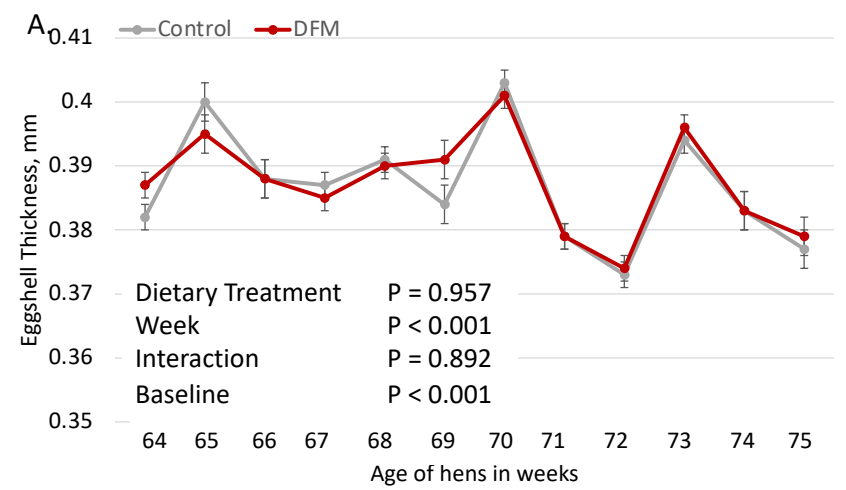

B. 48

C.
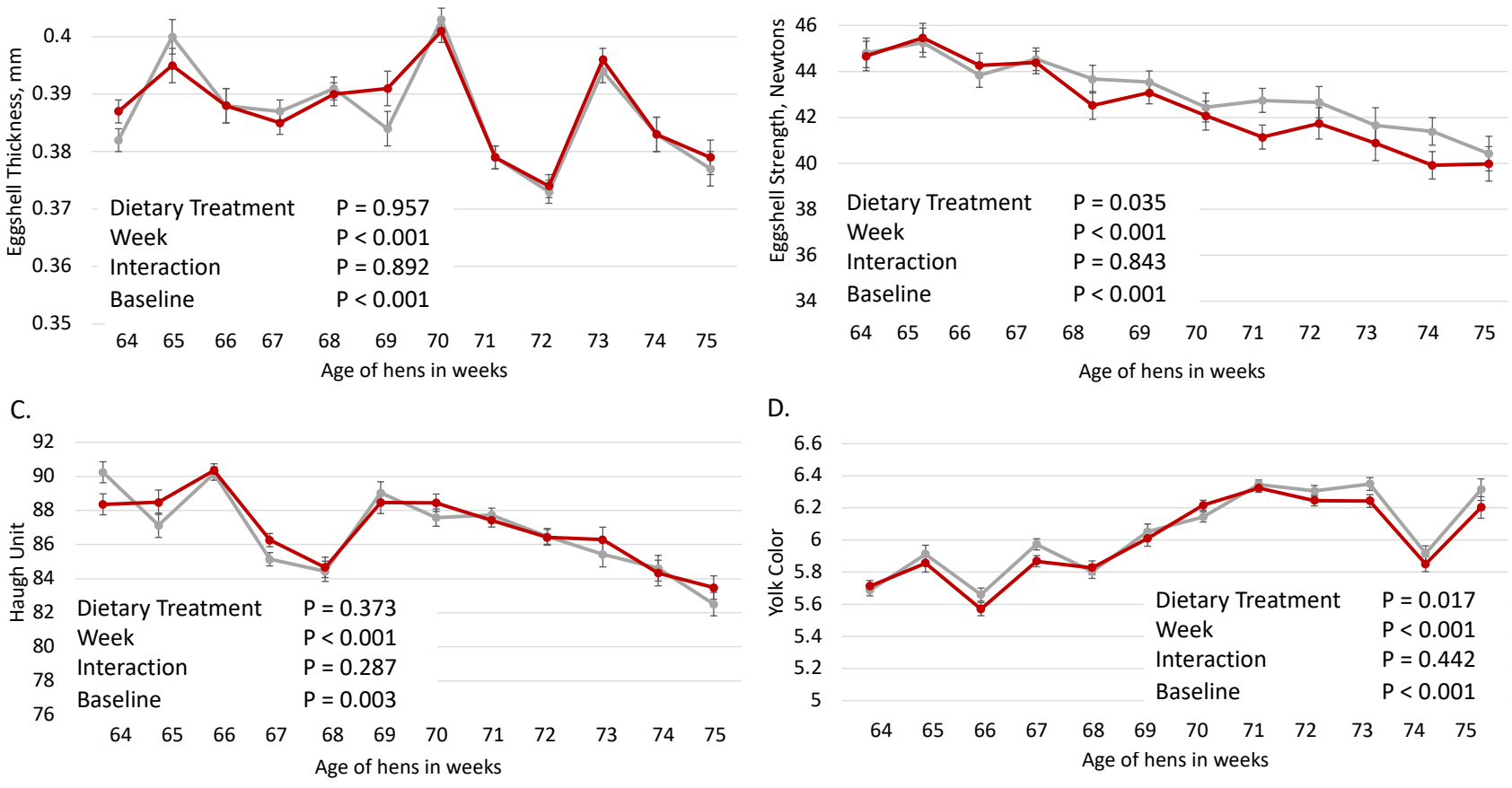

D.

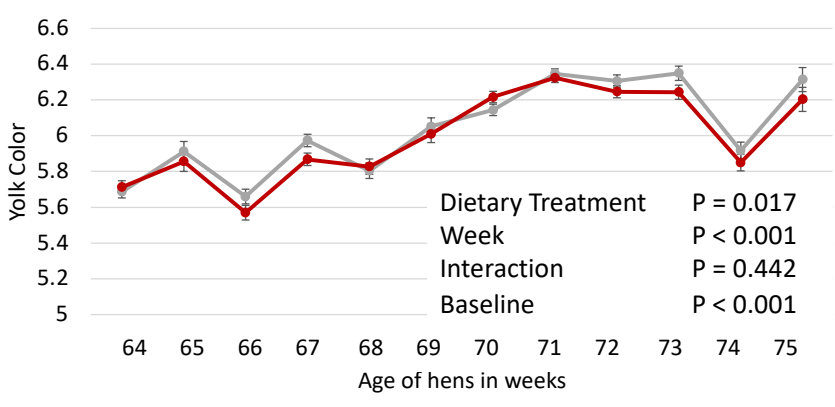

Figure 3. Egg quality measurements throughout the experimental period. A. Average weekly eggshell thickness measured in millimeters. B. Average weekly eggshell strength measured in Newtons. C. Average weekly Haugh unit. D. Average weekly yolk color. All parameters were measured using a DET6000. Grey lines represent hens fed the baseline or control diet. Red lines represent hens fed the DFM supplemented diet. 